\title{
SYSTEMIC LUPUS ERYTHEMATOSUS - DISEASE IMPACT ON PATIENTS
}

\author{
Želmíra Macejová', Michaele Záriková1, Mária Oetterová \\ 13rd Department of Internal Medicine, L. Pasteur University Hospital, Košice, Slovakia \\ 21st Department of Internal Medicine, L. Pasteur University Hospital, Košice, Slovakia
}

\section{SUMMARY}

Aim: Systemic lupus erythematosus (SLE) is an inflammatory autoimmune disease that can affect almost all organs of the body. Lupus is a non-curable disease and the treatment is based on symptom control by immunosuppressive and anti-inflammatory treatment. The disease itself as well as treatment-related adverse events have a significant negative impact on life expectancy and quality of life of patients. The aim of this study was to identify the impact of the disease on life of SLE patients.

Methods: Data were collected anonymously using a special questionnaire. The survey involved 76 patients with SLE, results were processed by conventional methods and descriptive statistic methods.

Results: The survey has confirmed the impact of SLE on professional activities i.e. up to $63 \%$ of patients are registered disabled, of which $46 \%$ are granted full disability pension. SLE negatively affects patients' career - up to $39 \%$ of SLE patients stated that they had to change a job due to their disease. SLE has a strong impact on everyday life of patients. SLE symptoms are significant even during the period of quiescence - predominantly fatigue, reduced physical activity, pain. The most influenced activities of daily living included sunbathing and more strenuous activities or sport. The fact that limitations in all monitored activities are present in more than $50 \%$ of patients is a serious finding. Treatment-related adverse events have negative impact on the quality of life in almost $70 \%$ of patients. The most frequent events include gastrointestinal symptoms, visual disturbance and osteoporosis.

Conclusion: SLE has a significant impact on the quality of life of patients and hinders them from leading everyday life at the level comparable to healthy population.

Key words: systemic lupus erythematosus, pain, fatique, impact of the disease

Address for correspondence: Ž. Macejová, 3rd Department of Internal Medicine, L. Pasteur University Hospital, tr. SNP 1, 04011 Košice, Slovakia. E-mail: zelmira.macejova@upjs.sk

\section{INTRODUCTION}

Systemic lupus erythematosus (SLE) is an inflammatory autoimmune disease that can affect almost all organs of the body and, as a consequence, this can lead to diverse clinical signs.

SLE affects a broad range of organ systems: skin, kidneys, central nervous system (CNS), heart, lungs, haematopoetic system, eyes and mucous membranes, gastrointestinal tract, reticuloendothelial and musculoskeletal system. Lupus is a noncurable disease, and the treatment is based on symptom control by immunosuppressive and anti-inflammatory treatment. The disease itself and disease-related consequences as well as treatmentrelated adverse events have a significant negative impact on life expectancy and quality of life of SLE patients $(1,2)$.

Relatively little information is available about the disease epidemiology, the impact of the disease on patients' quality of life, job position, disability, and their survival.

Available epidemiological studies estimated incidence of SLE in the range from 5 to 10 cases per 100,000 population. SLE is more prevalent among women than men. The number of newly diagnosed patients has been increasing recently. However, little is known about how the disease affects all aspects of patients' activities of daily living, including physical and mental health domain, and material domain.

The aim of this survey was to identify the impact of the disease on life of SLE patients.

The study was approved by the L. Pasteur University Hospital Ethical Committee in Košice, Slovakia.

\section{MATERIALS AND METHODS}

Data were collected anonymously using a special questionnaire. The questionnaire dealt with the impact of the disease on patient's daily life. No standardized questionnaire such as the SF-36 was used. All data were filled in by patients and sent to the project coordinators. The survey was a non-interventional survey and did not refer to any particular treatment. The survey was carried out at out-patient departments of rheumatology affiliated to the 1st and 3rd Department of Internal Medicine, University Hospital in Košice. No hypothesis was tested in the survey and the results were processed by conventional methods and descriptive statistic methods.

The survey involved 76 patients from the Slovak Republic examined during the period of July and August 2011. 


\section{RESULTS}

The group consisted of 76 patients ( $88 \%$ of women, $11 \%$ of men, in one case information on gender was not completed); the male to female ratio was 9:1, which corresponds exactly with the ratio in the published literature (2). The average age of patients was 42.6 years (standard deviation (SD) 12.5), the youngest patient was 20 and the oldest patient 79 years old. At the time of diagnosis, the average age was 29.3 years (SD 11.1, median 26 years), and mean time from onset of first symptom until diagnosis was 1.5 of a year. The average duration of disease was 13.1 years. Demographic distribution contributes to the well-known fact that women in productive age are mainly affected by SLE.

The impact of the disease on professional activities has confirmed that up to $63 \%$ of patients are registered disabled, of which $46 \%$ are granted full disability pension, $52 \%$ are granted partial disability pension; $2 \%$ of patients did not provide this information. Of patients with full disability pension, $27 \%$ are active (either working or studying); of patients with partial disability pension, $64 \%$ are active. Most patients, who are not registered as disabled, work full-time (Fig. 1).

SLE negatively affects patients' career - up to $39 \%$ of SLE patients stated that they had to change a job due to their disease, $24 \%$ of them had a job below their qualification level and $46 \%$ indicated lower income due to their disease. Only $12 \%$ of patients live on their own, $51 \%$ of patients live with a spouse and one third of patients live with their relatives or friends. The patients have higher than average education (37\% higher education, $40 \%$ secondary education with school leaving exam).

SLE has a strong impact on everyday life of patients. SLE symptoms are significant even during the period of quiescence - they include predominantly fatigue, reduced physical activity, pain, and cosmetic defects occurring in over $90 \%$ of patients (Fig. 2). Fatigue and pain are perceived by patients as substantial disturbance of normal functioning, hindering them from active organisation of their working and social life. Cosmetic defects are sensitively perceived mostly by female patients. This particularly concern rash, hair loss, flushing, and excessive hair in facial and neck area.

The least represented signs include psychological symptoms such as depression and memory disorders, however, they affect up to $50 \%$ of patients. In spite of lower occurrence they are perceived by patients as significantly affecting their daily functioning, including nervousness, depression, psychological exhaustion, mood change, and mental imbalance.

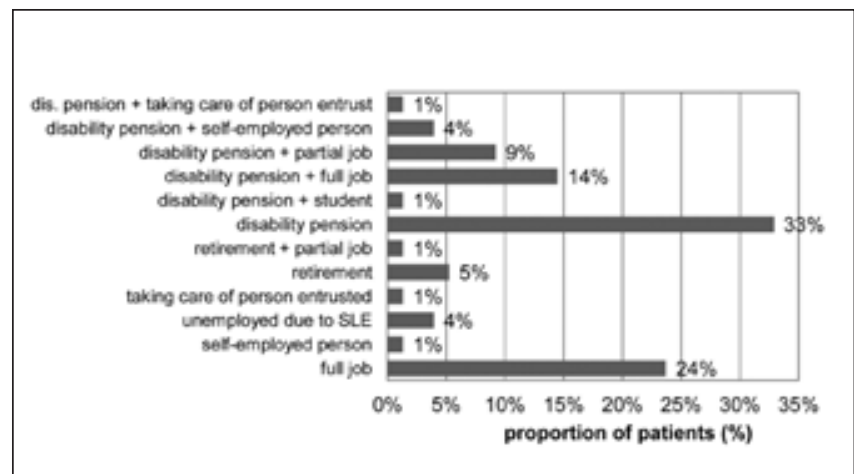

Fig. 1. Current job position.
The most frequent signs of the disease were at the same time evaluated by patients as the most intensive. During the quiescent period, fatigue occurs in almost $72 \%$ of patients and pain occurs in half of patients (49\%). Regardless pain frequency, the intensity is either significant $(42 \%)$ or mild $(50 \%)$; only $8 \%$ of patients do not feel pain during the quiescent period (Fig. 2).

At the time of data collection, $16 \%$ of all patients experienced deterioration, $66 \%$ were found to have stabilized SLE symptoms, and only $18 \%$ did not present any disease symptoms. Most of the patients consider the overall impact of SLE on the quality of life as either restrictive $(57 \%)$ or very restrictive $(21 \%)$. The most influenced areas include career (68\%) and social activities $(63 \%)$, and almost one third of patients indicate problems within their family and partnership, $30 \%$ of patients indicate limitation in "having a child or having more children".

The most influenced activities of daily living included sunbathing (during the summer season) and more strenuous activities or sport, including a significant proportion of patients who cannot carry out these activities at all (Fig. 3). The fact that limitations in all monitored activities are present in more than $50 \%$ of patients is a serious finding. That means that in almost all patients SLE significantly affects the possibility to lead everyday life comparable to matched healthy controls (Fig. 3).

In addition to the disease itself, treatment-related adverse events have negative impact on the quality of life in almost $70 \%$ of patients, with most of them experiencing several adverse events at a time. The most frequent events include gastrointestinal symptoms (32\%) followed by a wide spectrum of serious health complications such as visual disturbance, osteoporosis, etc. (Table 1). Many patients complain of abdominal pain, nausea, weight

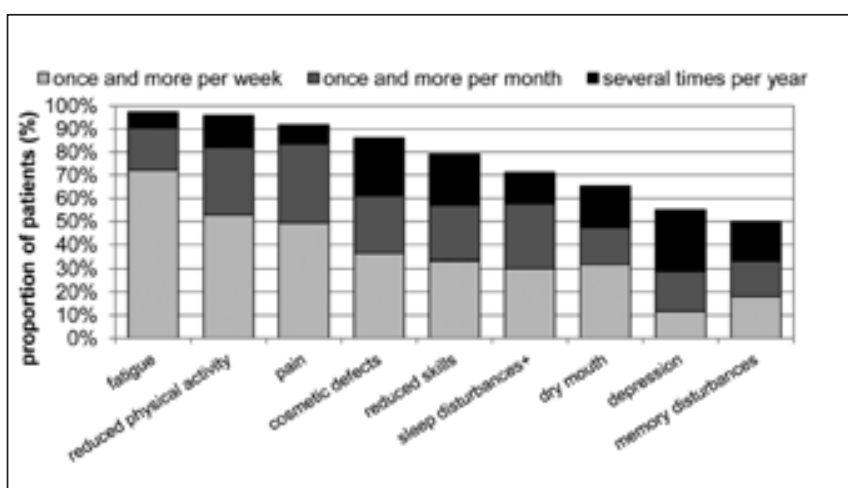

Fig. 2. SLE symptom frequency during the quiescent period.

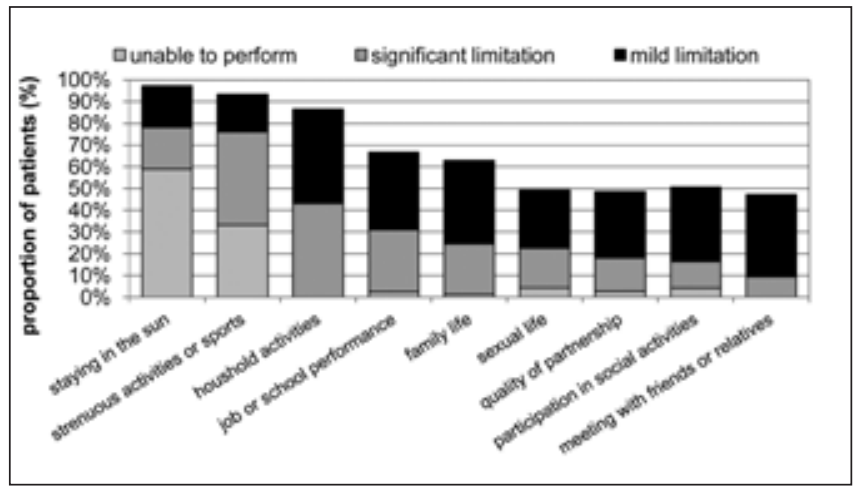

Fig. 3. Limitations in daily activities. 
gain, gastric ulcer, and overweight. Other frequent adverse events include skin problems - rash, flushing, expressive sweating, itching, hair loss, hirsutism in facial and neck area. These do not present a significant health risk; however, they are often identified as the most visible symptoms, confirming the disease stigmatization, mainly in women. In addition to treatment during flares, treatment needed in quiescent period is perceived as burden by many patients, while in acute flares the drugs are inevitable (Table 1).

Necessity of frequent doctor's visits, disease-associated hospitalizations and disease-associated disability significantly affect the quality of everyday life of patients. In the past 12 months, 38\% of patients were hospitalized for SLE with average of 0.96 hospitalisations per patient. In the past calendar year a sick-leave was reported in $51 \%$ of working patients with average of 1.0 sick-leave per patient.

Regarding patient information, only two thirds of all patients consider information about the disease and treatment sufficient. A treating physician and internet are among the most common information sources, while only very few patients obtain information from other sources - magazines, internet, or family and friends.

\section{DISCUSSION}

The survey shows that lupus predominantly affects women in productive age. More than half of the patients regard the overall impact of SLE as restrictive (57\%) and one fifth as very restrictive $(21 \%)$. The disability rate is high, almost $63 \%$ of patients are registered disabled (46\% are granted full disability pension, and $52 \%$ are granted partial disability pension). Our results are similar with the LUMINA study. Analysis of 273 patients with early

Table 1. SLE treatment-related adverse events

\begin{tabular}{|l|c|c|}
\hline Adverse events & $\begin{array}{c}\text { Number } \\
\text { of patients }\end{array}$ & $\begin{array}{c}\text { Proportion } \\
\text { of patients }\end{array}$ \\
\hline Gl symptoms & 24 & $32 \%$ \\
\hline Vessel disorders & 13 & $17 \%$ \\
\hline Visual problems & 14 & $18 \%$ \\
\hline Weight gain & 13 & $17 \%$ \\
\hline Skin disorders & 11 & $14 \%$ \\
\hline Osteoporosis & 10 & $13 \%$ \\
\hline Alopecia & 10 & $13 \%$ \\
\hline Blood pressure disturbances & 10 & $13 \%$ \\
\hline Psychical problems & 10 & $13 \%$ \\
\hline Pain & 7 & $9 \%$ \\
\hline Palpitation & 5 & $7 \%$ \\
\hline Kidney disorders & 5 & $7 \%$ \\
\hline Fatique & 5 & $7 \%$ \\
\hline Infections & 5 & $7 \%$ \\
\hline Hirsutism & 4 & $5 \%$ \\
\hline Oedema & 4 & $5 \%$ \\
\hline Striae & 3 & $4 \%$ \\
\hline Muscle weakness & 2 & $3 \%$ \\
\hline Inflammation & 2 & $3 \%$ \\
\hline Lipid disturbances & & \\
\hline & $5 \%$ & $7 \%$ \\
\hline
\end{tabular}

SLE employed at enrolment, 19\% were unable to work because of disability after a 5-year follow-up (3). Yelin et al. reported a steadily increasing proportion of SLE patients unable to work with increasing time of the disease duration, $15 \%$ at 5 years to $63 \%$ at 20 years of duration (4).

The disease gives rise to a wide range of clinical manifestations including the quiescent period that most often involve fatigue, reduced physical activity, and mild or severe pain. The symptoms like fatigue, pain, and musculoskeletal distress were also most frequently reported in other studies (5). Patients suffer from multiple symptoms concurrently and even the least frequent symptoms such as depression or memory disturbance occur in more than $50 \%$ of patients in the period of quiescence. Relationship between glucocorticoid dose and depression was observed also in study of Choi and co-authors (6). In addition to the disease itself, patients are negatively affected by treatment-related adverse events reported in almost $70 \%$ of patients, and most of them experience several adverse events concurrently.

The disease affects many aspects of patient's life (7). Patients perceive disturbance in the area of job performance, career building $(68 \%)$ and social activities $(63 \%)$ as the most significant, leading to social isolation and frustration resulting from inability to perform activities of daily living that used to be common prior to the disease. The disease affects the area of human relationships, partnership and family life. Problems within family and partnership were recorded in approximately one third of patients.

The most influenced ordinary activities included sunbathing and more strenuous activities or sport, including a significant proportion of patients who cannot carry out these activities at all. Limitations in all monitored activities are present in more than 50\% of patients. Finally, we can conclude that SLE has a significant impact on the quality of life of patients and hinders them from leading everyday life at the level comparable to healthy population.

\section{REFERENCES}

1. Alarcón GS, McGwin G Jr, Uribe A, Friedman AW, Roseman JM, Fessler BJ, et al. Systemic lupus erythematosus in a multiethnic lupus cohort (LUMINA). XVII. Predictors of self-reported health-related quality of life early in the disease course. Arthritis Rheum. 2004 Jun 15;51(3):465-74.

2. Almehed K, Carlsten H, Forsblad-d'Elia H. Health-related quality of life in systemic lupus erythematosus and its association with disease and work disability. Scand J Rheumatol. 2010;39(1):58-62.

3. Bertoli AM, Fernández M, Alarcón GS, Vilá LM, Reveille JD. Systemic lupus erythematosus in a multiethnic US cohort LUMINA (XLI): factors predictive of self-reported work disability. Ann Rheum Dis. 2007 Jan;66(1):12-7.

4. Yelin E, Trupin L, Katz P, Criswell L, Yazdany J, Gillis J, et al. Work dynamics among persons with systemic lupus erythematosus. Arthritis Rheum. 2007 Feb 15;57(1):56-63.

5. Pettersson S, Lövgren M, Eriksson LE, Moberg C, Svenungsson E, Gunnarsson I, et al. An exploration of patient-reported symptoms in systemic lupus erythematosus and the relationship to health-related quality of life. Scand J Rheumatol. 2012 Oct;41(5):383-90.

6. Choi ST, Kang JI, Park IH, Lee YW, Song JS, Park YB, et al. Subscale analysis of quality of life in patients with systemic lupus erythematosus: association with depression, fatigue, disease activity and damage. Clin Exp Rheumatol. 2012 Sep-Oct;30(5):665-72.

7. Campbell R Jr, Cooper GS, Gilkeson GS. Two aspects of the clinical and humanistic burden of systemic lupus erythematosus: mortality risk and quality of life early in the course of disease. Arthritis Rheum. 2008 Apr $15 ; 59(4): 458-64$ 